\title{
A nomogram to predict the overall survival of patients with symptomatic extensive-stage small cell lung cancer treated with thoracic radiotherapy
}

\author{
Xun Yuan ${ }^{1 \#}$, Zhiqin Zheng, ${ }^{2,3 \#}$, Fangfang Liu ${ }^{1}$, Yuan Gao ${ }^{1}$, Wenhui Zhang ${ }^{4}$, Rossana Berardi ${ }^{5}$, \\ Pranshu Mohindra ${ }^{6}$, Zhengfei Zhu ${ }^{2,7,8}$, Jie Lin ${ }^{4}$, Qian Chu ${ }^{1}$
}

${ }^{1}$ Department of Oncology, Tongji Hospital of Tongji Medical College, Huazhong University of Science and Technology, Wuhan, China; ${ }^{2}$ Department of Radiation Oncology, Fudan University Shanghai Cancer Center, Shanghai, China; ${ }^{3}$ Department of Radiation Oncology, Minhang Branch Hospital, Fudan University Shanghai Cancer Center, Shanghai, China; ${ }^{4}$ Department of Oncology, The Second Affiliated Hospital of Kunming Medical University, Kunming, China; ${ }^{5}$ Clinica Oncologica, Università Politecnica delle Marche, Azienda Ospedaliero-Universitaria Ospedali Riuniti Umberto I, GM Lancisi, G Salesi di Ancona, Italy; ' Department of Radiation Oncology, University of Maryland School of Medicine, Baltimore, MD, USA; ${ }^{7}$ Department of Oncology, Shanghai Medical College, Fudan University, Shanghai, China; ${ }^{8}$ Institute of Thoracic Oncology, Fudan University, Shanghai, China

Contributions: (I) Conception and design: X Yuan, Q Chu; (II) Administrative support: R Berardi, P Mohindra; (III) Provision of study materials or patients: Z Zheng, F Liu; (IV) Collection and assembly of data: Y Gao, W Zhang; (V) Data analysis and interpretation: Z Zhu, J Lin; (VI) Manuscript writing: All authors; (VII) Final approval of manuscript: All authors.

"These authors contributed equally to this work.

Correspondence to: Qian Chu, MD, PhD. Department of Oncology, Tongji Hospital of Tongji Medical College, Huazhong University of Science and Technology, 1095 Jiefang Avenue, Wuhan 430030, China. Email: qianchu@tjh.tjmu.edu.cn; Jie Lin, MD. Department of Oncology, the Second Affiliated Hospital of Kunming Medical University, 374 Dianmian Avenue, Wuhua District, Kunming 650101, China. Email: linjieshi@126.com; Zhengfei Zhu, MD. Department of Radiation Oncology, Fudan University Shanghai Cancer Center, 270 Dong An Road, Shanghai 200032 China. Email: fuscczzf@163.com.

Background: Small cell lung cancer (SCLC) makes up 13\% of lung malignancies. Only one-third of SCLC patients received their diagnosis at the limited stage. Treatment for symptomatic extensive-stage (ES) SCLC with persistent intrathoracic disease is still controversial. The present research aimed to analyze the impact of palliative thoracic radiotherapy (TRT) as a treatment for this patient group and build a prognostic nomogram.

Methods: In this retrospective, multi-center study, we analyzed 120 patients with ES-SCLC and a World Health Organization performance status of 1-2 who were diagnosed between March 2014 and September 2019. A nomogram was formulated to predict the patients' 1- and 2-year overall survival (OS).

Results: The study cohort had a median age of 62 years, and males accounted for $85 \%$ of enrollees. A significant extension was observed in the median OS in the TRT group compared to the no TRT group $(\mathrm{P}<0.001)$. When the patients were stratified by TRT dose, no significant differences in OS were noted $(\mathrm{P}=0.530)$. However, higher levels of inflammatory markers prior to TRT were associated with a shorter OS (neutrophil-to-lymphocyte ratio, $\mathrm{P}=0.002$; platelet/lymphocyte ratio, $\mathrm{P}=0.023$ ). The nomogram's Harrell's concordance (C)-statistic reached 0.70 , and the calibration curve analysis revealed goodness of fit.

Conclusions: The neutrophil-to-lymphocyte ratio is an independent factor predicting survival in ESSCLC patients treated with palliative TRT. Our nomogram, which incorporates immunological markers, has higher accuracy than existing models for the prediction of individuals' chances of survival, and it could be a significant tool for clinicians in the development of tailored therapeutic strategies.

Keywords: Extensive stage small cell lung cancer (ES-SCLC); palliative thoracic radiotherapy (TRT); neutrophilto-lymphocyte ratio (NLR); platelet/lymphocyte ratio (PLR); nomogram 
Submitted Sep 24, 2020. Accepted for publication Apr 14, 2021.

doi: $10.21037 /$ tlcr-21-211

View this article at: http://dx.doi.org/10.21037/tlcr-21-211

\section{Introduction}

With a 5 -year survival rate of $<10 \%$, small cell lung cancer (SCLC) is a malignancy which exhibits a high level of aggression and has the characteristics of growing rapidly and metastasizing early. SCLC comprises around $13 \%$ of all lung cancers and claims 250,000 lives globally each year $(1,2)$. One-third of SCLC cases are classified as limited stage (LS), and the rest are diagnosed as extensive-stage SCLC (ES-SCLC) (3). Common presenting signs and symptoms of ES-SCLC include the following: shortness of breath, cough, bone pain, weight loss, fatigue and neurologic dysfunction. The standard first-line treatment strategy for ES-SCLC is 4-6 cycles of cisplatin-based doublet chemotherapy with immunotherapy $(4,5)$. However, intrathoracic disease progression remains a major problem and the overall survival is not satisfactory. Consolidative thoracic radiotherapy (TRT) has shown a survival benefit for patients with ES-SCLC in several prospective and retrospective studies. For instance, a single-institution randomized study in which 210 patients were designated at random to receive TRT (54 Gy in 36 fractions given twice per day) with concurrent chemotherapy $v s$. chemotherapy alone, suggested a benefit of adding TRT in terms of median survival (17 vs. 11 months, $\mathrm{P}=0.041$ ) (6). In the phase 3 Chest Radiotherapy Extensive-Stage Small Cell Lung Cancer Trial (CREST), TRT (a dose of 30 Gy in 10 fractions) significantly improved 2-year overall survival (OS) and progression-free survival (PFS), although these did not constitute the study's primary endpoints (7). As such, the true benefit of TRT for patients with ES-SCLC and a World Health Organization (WHO) performance status (PS) of 1-2 and the optimal dose of palliative TRT are unknown. The identification of markers and models that could potentially predict patients' prognoses also massively impacts the creation of clinical therapeutic strategies.

Systemic immune and inflammatory status have pivotal involvement in tumorigenesis, cancer progression, and responses to therapies (8). Evidence exists suggesting close associations between systemic inflammatory response markers, such as the neutrophil-to-lymphocyte ratio (NLR), the platelet/lymphocyte ratio (PLR), and lactate dehydrogenase (LDH), and SCLC prognosis $(9,10)$. The NLR is a simple and cost-effective biomarker that can be used as part of routine laboratory evaluation, even in resource-limited settings $(11,12)$. However, currently, the relevance of this pretreatment inflammatory biomarker to the prognosis of patients receiving palliative TRT for ESSCLC remains poorly understood.

The present work aimed to investigate the benefit of palliative TRT in ES-SCLC, as well as the prognostic relevance of NLR as a pretreatment inflammatory biomarker with regard to OS in patients with ES-SCLC receiving palliative TRT.

We present the following article in accordance with the STROBE reporting checklist (available at http://dx.doi. org/10.21037/tlcr-21-211).

\section{Methods}

\section{Patients}

We conducted this retrospective study of patients treated between March 2014 and September 2019 at 3 hospitals: Tongji Hospital of Tongji Medical College, Fudan University Shanghai Cancer Center, and Fujian Cancer Hospital. Patients meeting the following criteria were included in the study: (I) pathologically confirmed SCLC; (II) newly diagnosed ES-SCLC (namely, disease extending beyond the hemithorax, hilar, mediastinal, and supraclavicular nodes); (III) aged $\geq 18$ years; (IV) complete clinical, laboratory, imaging, and follow-up data; (V) no previous anticancer therapies; (VI) WHO PS of 1-2 with hemoptysis, blood in sputum, face and neck edema, wheezing, chest pain, headache, or persistent cough. The exclusion criteria included evidenced infection, bone marrow, hematological, or autoimmune disease. Early mortality (i.e., occurring in the 3 months following diagnosis) was also an exclusion criterion, as it was likely to have resulted in the patient not being considered for the entire course of TRT and chemotherapy.

Eligible patients were allocated to either the noTRT group or the TRT with sequential or concurrent chemoradiotherapy group. The median dose in the TRT cohort (dose ranging from 30 to $60 \mathrm{~Gy}$ ) was 50 Gy using intensity modulated radiation therapy (IMRT), with most patients receiving $45 \mathrm{~Gy} / 30 \mathrm{f}$ or $60 \mathrm{~Gy} / 30 \mathrm{f}$ (13). Chemotherapy consisted of 4-6 cycles of cisplatin plus 
etoposide. Additionally, some patients in both groups received radiation to target lesions in the brain or metastatic lesions in the bone. Patients were typically followed up at 30 days after treatment initiation, then at 3-month intervals for the first 2 years, and at 6-month intervals thereafter.

This research was approved by the institutional review board of Tongji Hospital of Tongji Medical College, Huazhong University of Science and Technology (TJIRB20190404). All procedures were conducted in accordance with the principles of the Declaration of Helsinki (as revised in 2013). Individual patient information was protected and has not been shown. Individual consent for this retrospective analysis was waived.

\section{Data collection}

Histological diagnosis was made according to the WHO classification version 7. The $8^{\text {th }}$ edition of the American Joint Cancer Committee (AJCC) tumor-node-metastasis (TNM) classification was used for disease staging, with stage IV constituting ES-SCLC. Patient characteristics, such as age (<60 vs. $\geq 60$ years), sex, smoking history, and details of treatment strategies were collected for the analysis. In the week preceding TRT initiation, patients underwent a full blood count and routine biochemistry test. The NLR was obtained by dividing the neutrophil count by the lymphocyte count, and the PLR was obtained by dividing the platelet count by the lymphocyte count. Receiver operating characteristic (ROC) curve analysis was used to determine the best cutoff values for pretreatment NLR and PLR. Follow-up was performed until June 2020. OS was calculated as the interval commencing on the date of diagnosis or treatment initiation to the date of death or the final follow-up appointment. The symptom relief rate was defined as the proportion of patients who attained symptom relief.

\section{Statistical analysis}

Summary statistics were reported for discrete variables. For the OS evaluation of the ES-SCLC patients, Kaplan-Meier (KM) curves were drawn to express the cumulative survival probability, and the log-rank test was used to compare OS between groups. We conducted univariate and multivariate analysis to identify prognostic factors, including clinicopathological variables and pretreatment hematologic markers, by adopting the Cox proportional hazards model. We also plotted a nomogram to predict 1 - and 2-year
OS, and determined the ability of the model to accurately predict prognosis by employing Harrell's concordance index (C-index). Calibration curve analysis was carried out to evaluate the model's goodness of fit. We internally validated the predictive accuracy of the model and avoided overfitting by using 1,000 bootstrap resamples. All $\mathrm{P}$ values were 2 -tailed, with $\mathrm{P}<0.05$ being statistically significant. All statistical analyses were performed with $\mathrm{R}$ language, version 4.0.2 with the rms and survival libraries (The R Foundation for Statistical Computing, Vienna, Austria, http://www. r-project.org/).

\section{Results}

\section{Patient characteristics}

The baseline characteristic information for each group was similar (Table 1). In the entire cohort, the median diagnostic age was 62 years (30-77 years), and males were in the majority (85\%). Metastasis to the brain, liver, and bones was reported in $44.0 \%, 26.4 \%$, and $36.3 \%$ of the study population, respectively. The chemotherapeutic regimens of 114 (95\%) patients combined platinum with etoposide. A total of 6 patients did not receive or stopped chemotherapy ( 2 cases due to refusal, and 4 cases due to treatmentrelated toxic effects). In the chemotherapy cohort, the disease control rate reached $73.5 \%$. The median TRT dose received by patients was $50 \mathrm{~Gy} ; 31$ and 40 patients were given $\leq 50$ and $>50 \mathrm{~Gy}$, respectively. Both groups had a similar symptom relief rate $(90 \%)$.

In line with the clinical criteria devised by the Department of Clinical Laboratory at Tong Hospital of Tongji Medical College, a value of $225 \mathrm{U} / \mathrm{L}$ was used to differentiate high and low levels of serum biomarker LDH. The cutoff values for NLR and PLR in the survival outcome prediction of ES-SCLC served as a basis for dichotomizing NLR and PLR, respectively. We took the values showing the best relative risk coefficients at 5 and 210 as the optimal cutoffs for NLR and PLR. The areas under the timedependent ROC curves (AUCs) for the dichotomized NLR and PLR were also calculated. PLR was the indicator with the greatest AUC value (0.741), which suggested that it possessed the highest predictive value (Figure 1).

\section{Survival}

The median OS for patients who received radiotherapy was 19 months compared to 14 months for patients without 
Table 1 Clinicopathological characteristics of ES-SCLC patients $(\mathrm{n}=120)$

\begin{tabular}{|c|c|c|}
\hline Characteristics & $\begin{array}{l}\text { No-TRT group } \\
\qquad(n=49)\end{array}$ & $\begin{array}{l}\text { TRT group } \\
\quad(n=71)\end{array}$ \\
\hline \multicolumn{3}{|c|}{ Age (years) (n [\%]) } \\
\hline$<60$ & 17 & 30 \\
\hline$\geq 60$ & 32 & 41 \\
\hline \multicolumn{3}{|l|}{ Sex [n (\%)] } \\
\hline Female & 7 & 11 \\
\hline Male & 42 & 60 \\
\hline \multicolumn{3}{|l|}{ Smoking } \\
\hline Former & 27 & 36 \\
\hline Current & 22 & 35 \\
\hline \multicolumn{3}{|l|}{ Chemotherapy } \\
\hline No & 6 & 0 \\
\hline Yes & 43 & 71 \\
\hline \multicolumn{3}{|c|}{ Brain radiotherapy } \\
\hline No & 25 & 42 \\
\hline Yes & 24 & 29 \\
\hline \multicolumn{3}{|c|}{ Bone radiotherapy } \\
\hline No & 44 & 67 \\
\hline Yes & 5 & 4 \\
\hline \multicolumn{3}{|l|}{ Immunotherapy } \\
\hline No & 46 & 67 \\
\hline Yes & 3 & 4 \\
\hline
\end{tabular}

ES-SCLC, extensive-stage small cell lung cancer.

radiotherapy [hazard ratio $(\mathrm{HR})=0.443,95 \%$ confidence interval (CI): $0.247-0.796, \mathrm{P}<0.001$ ] (Figure 2). In the TRT group, $52 \%$ and $39 \%$ of patients experienced intrathoracic recurrence and brain progression, respectively. We further partitioned dose groups into low- and high-dose categories; however, high-dose radiotherapy did not show significant survival advantages over low-dose radiotherapy $(\mathrm{P}=0.530)$ (Table 2).

During a median 13-month follow-up, the KM analysis revealed a low NLR and PLR to bear significance in the prediction of prolonged survival (Figure 3). When age, sex, smoking history, chemotherapy sequence, and radiotherapy dose were adjusted for in the multivariable analysis, NLR remained an independent predictor (Table 2). Next, a

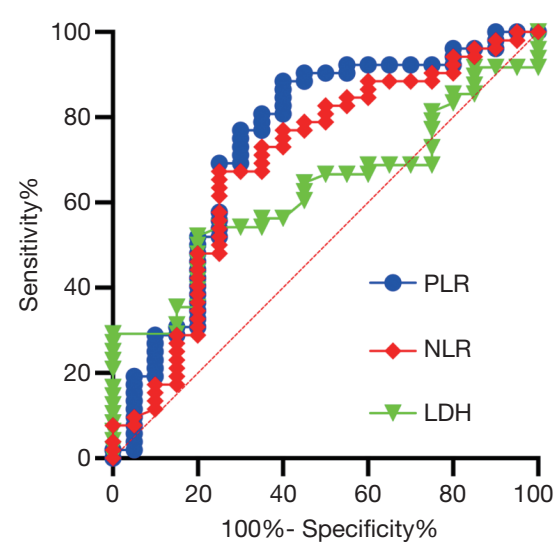

Figure 1 Survival outcomes of ES-SCLC patients receiving thoracic radiotherapy compared to patients without radiotherapy. ES-SCLC, extensive-stage small cell lung cancer.

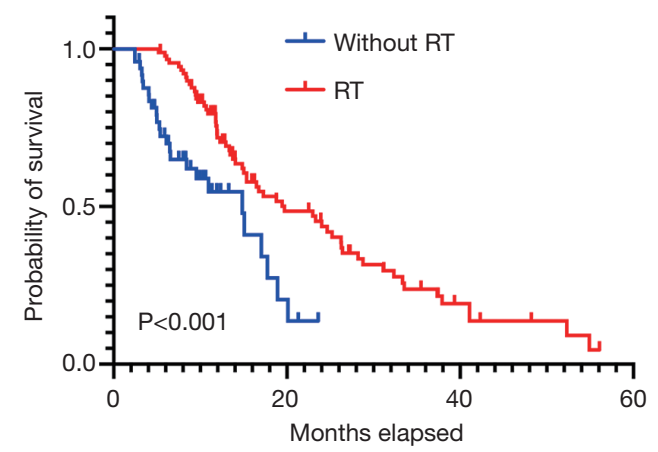

Figure 2 ROC curve analysis of different markers to predict patients' survival status. ROC, receiver operating characteristic.

nomogram incorporating variables such as age, sex, sequence of chemotherapy and radiation, radiotherapy dose, and symptoms after TRT was created to evaluate the prognostic relevance of these clinical parameters in ES-SCLC treated with TRT (Figure 4). The Harrell's C-index for the clinicopathologic multivariate model was 0.63 . Of note, when NLR and PLR were incorporated into the clinicopathologic model, a significant improvement was observed in the prognostic accuracy, with the Harrell's C-index rising to 0.70 . The model exhibited moderate discriminative ability and was adequately calibrated (Figure S1).

\section{Discussion}

SCLC is among the most malignant of lung cancers worldwide. At present, standard treatment for patients 
Table 2 Univariate and Multivariate Cox regression analyses of multiple variables for OS in ES-SCLC treated with palliative thoracic radiotherapy $(\mathrm{n}=71)$

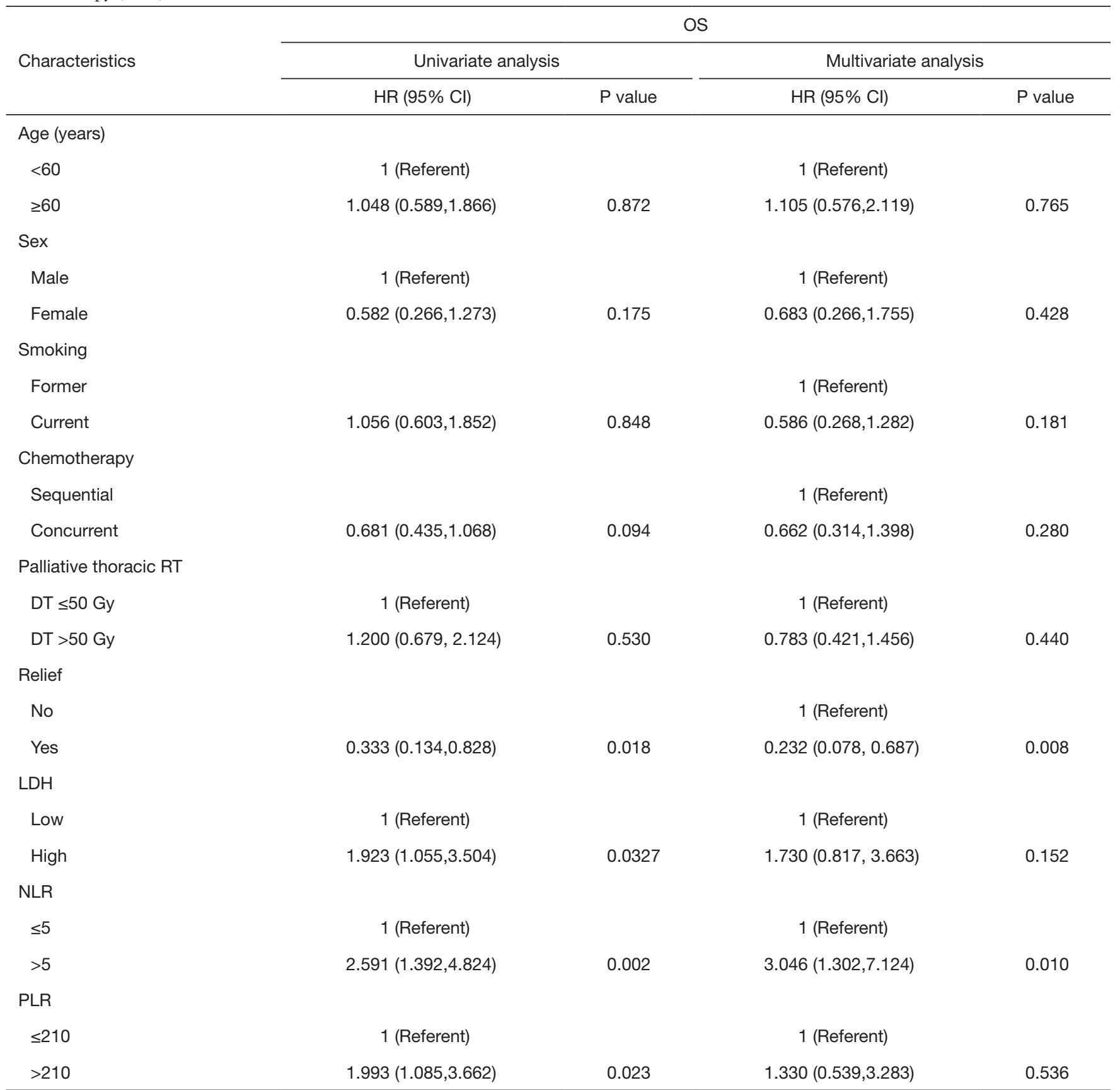

ES-SCLC, extensive-stage small cell lung cancer; OS, overall survival; Cl, confidence interval; HR, hazard ratio; LDH, lactate dehydrogenase; NLR, neutrophil-to-lymphocyte ratio; PLR, platelet/lymphocyte ratio.

with ES-SCLC consists of 4-6 cycles of platinumbased chemotherapy with immunotherapy. However, approximately $20 \%$ of those diagnosed with SCLC are not offered chemotherapy (14). The chance of being offered treatment decreased with PS. A recent study of ES-SCLC patients aged $\geq 70$ years reported a 7.8- and 0.6-month median OS among participants who received chemotherapy and no treatment, respectively (15); those who received 

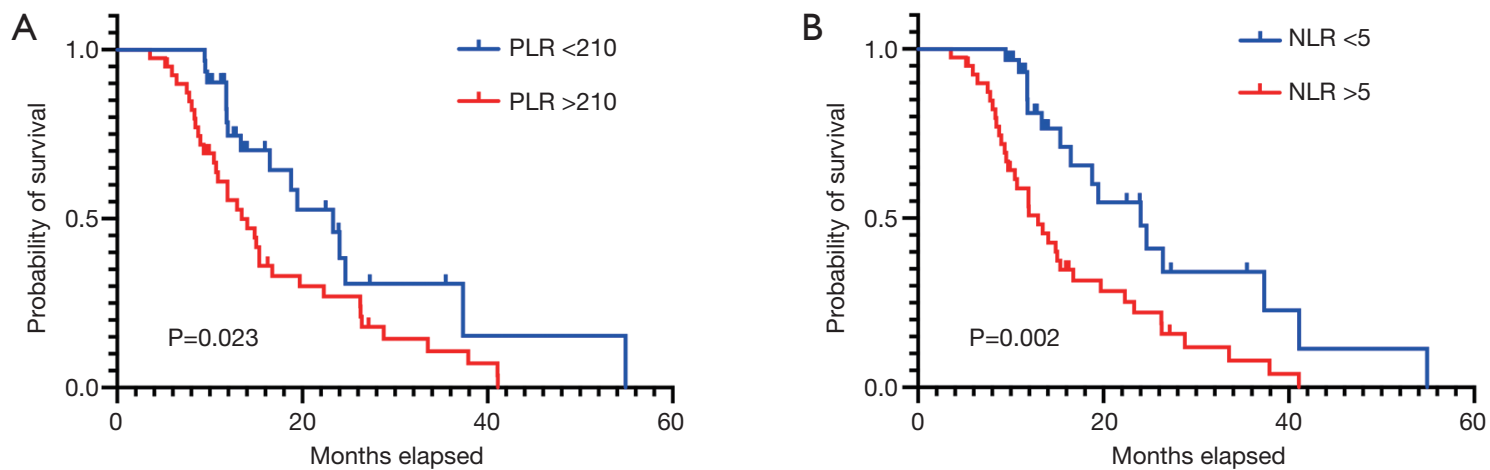

Figure 3 Prognostic analysis of serum indicators in ES-SCLC. (A) Kaplan-Meier curve for OS showing a significant association between a high NLR and a low OS rate in ES-SCLC. (B) Kaplan-Meier curve for OS showing a significant association between a high PLR and a low OS rate in ES-SCLC. ES-SCLC, extensive-stage small cell lung cancer; OS, overall survival; PLR, platelet/lymphocyte ratio.

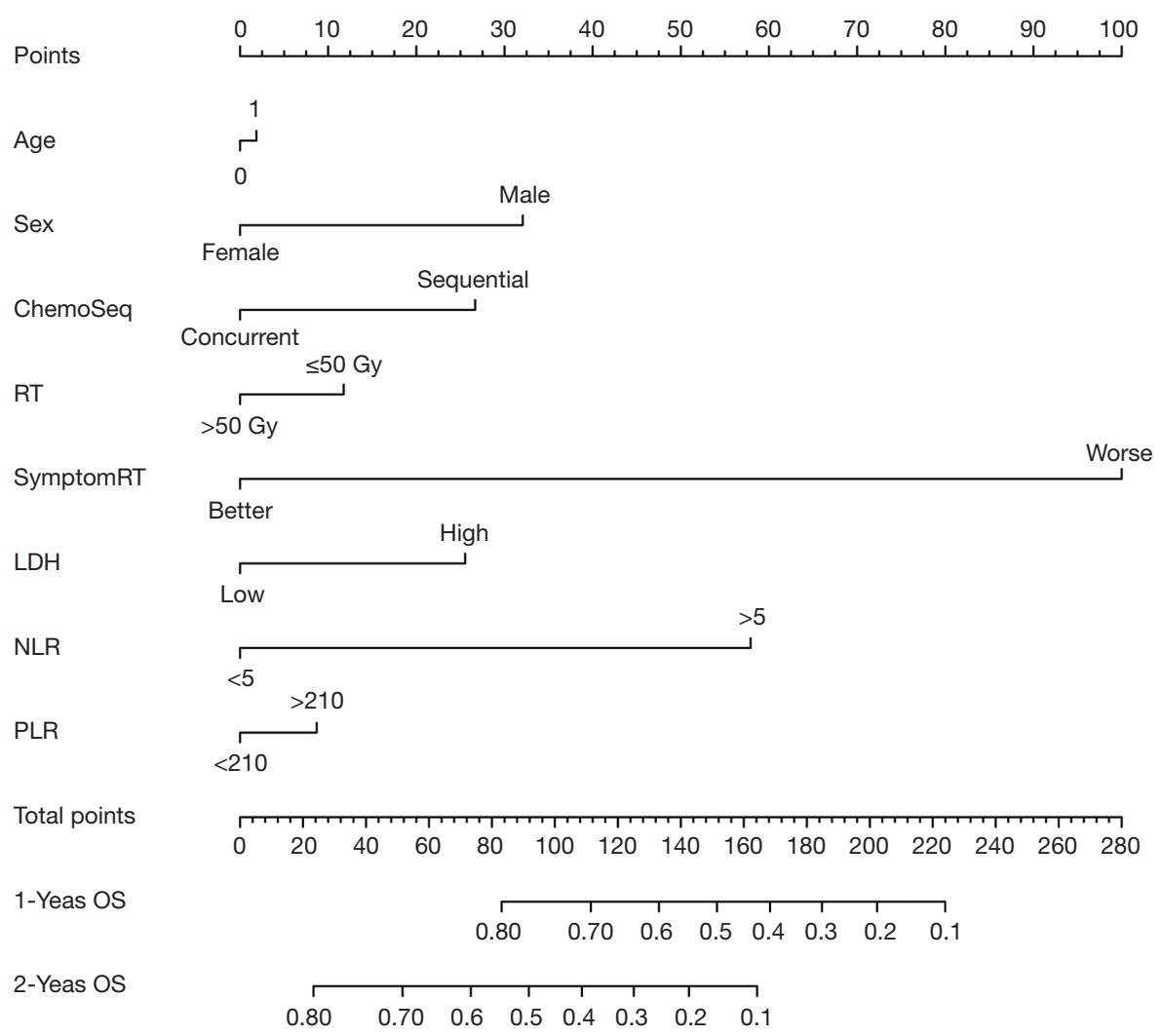

Figure 4 Nomogram predicting 1- and 2-year survival in ES-SCLC patients. The nomogram is applied by summing up the points identified on the points scale for each variable. The total projected on the bottom scale gives an indication of the patient's survival probability after 1 and 2 years. ES-SCLC, extensive-stage small cell lung cancer; ChemoSeq, sequence of chemotherapy and radiation; RT, radiation; SymptomRT, symptom after radiation; NLR, neutrophil-to-lymphocyte ratio; PLR, platelet/lymphocyte ratio.

chemotherapy had substantially increased OS. In the present research, we examined data from a large group of patients enrolled from multiple institutions, all of whom had a PS of 1-2, and performed a comparison of the outcomes of patients who received either chemoradiotherapy or only chemotherapy. Compared to the no-TRT group 
(14 months), chemoradiotherapy led to a substantial increase in OS (19 months). These findings are largely consistent with those of previous retrospective and randomized research on TRT, which have suggested the survival benefit of TRT concurrent with chemotherapy for systematic ES-SCLC. Apart from survival benefit, TRT was associated with symptomatic chest recurrences in only $13 \%$ of patients. In a clinical trial, 32 ES-SCLC patients who attaining an objective response to chemotherapy were enrolled to define the rate of symptomatic chest failures after undergoing post-chemotherapy consolidation TRT (16). Of the 19 intrathoracic recurrences, only 5 were symptomatic. This indicated that TRT could not only improve local control but also provide an excellent symptomatic control in the irradiated chest region for ESSCLC patients.

Recurrent intrathoracic disease is observed in more than $40 \%$ of patients who receive TRT, indicating that increasing the radiation dose might be more efficacious (16). Hasan et al. organized ES-SCLC patients into low-dose (30-39 Gy), intermediate-dose (40-59 Gy), and high-dose (59.4-75 Gy) TRT groups, and the median OS reached 10.7, 12.9, and 14.9 months, respectively $(\mathrm{P}<0.001)(17)$. However, in the present study, high-dose radiotherapy did not show a significant survival advantage over lowdose radiotherapy. One of the reasons for this may be that disease progression occurred in areas other than the thorax and the brain in many patients, in spite of the use of TRT; thus, the additional dose of radiotherapy to thoracic disease sites may have been futile and only increased the toxicity in frail patients. Professor Han ever conducted a study to evaluate the clinical outcomes of 244 ES-SCLC patients who received TRT at different times and fractionations and tried to explore practice patterns for the radiation time and fractionation (18). Patients who received TRT within 6 cycles of chemotherapy and hyperfractionated radiotherapy (45 Gy in 30 fractions) had relatively long OS. In this study, we also find that concurrent TRT had a tendency to prolong survival for ES-SCLC patients. Further studies are warranted to clarify this hypothesis.

The recent progress with immune checkpoint inhibitors has seen a survival extension for patients with ES-SCLC. Despite inflammation and immunity being recognized as participants in ES-SCLC development, the disease has yet to be fully elucidated from an immunological perspective. The present work investigated the correlations of pretreatment variables, such as LDH and inflammatory markers, with survival of ES-SCLC. The enzyme LDH, which facilitates lactic acid production in the glycolytic pathway, frequently serves as an indicator for the monitoring and assessment of the disease state. LDH has been detected in various damaged tissues and malignancies (19-21). However, like that of the NLR and the PLR, the predictive value of LDH for ES-SCLC patients receiving palliative TRT remains controversial (10). Here, we found that a low NLR was indicative of a favorable survival outcome, whereas we failed to identify an independent survival association for PLR or LDH. In line with previously reported research on other cancers, our study evidenced the utility of the NLR as an indicator predicting ES-SCLC prognosis (22-25). The potential of the NLR to serve as a predictive biomarker of immunotherapeutic efficacy in ES-SCLC is deserving of more research attention and will be evaluated in future experiences that include use of immunotherapy. Until such reports are available in the published literature, the current analysis represents 1 of the largest of its kind in the pre-immunotherapy era. Importantly, we created a nomogram which incorporates NLR, PLR, LDH, and other clinicopathologic factors to predict the OS of patients with ES-SCLC, and subjected it to internal validation. Concurrent chemoradiotherapy and higher dose demonstrated superiority in terms of OS. Patients with higher total points of the nomogram might benefit from concurrent chemoradiotherapy and higher dose. We believe our nomogram will facilitate tailored OS prognostication for patients and serve as a clinical tool to guide treatment-related decisions. We will further define the high-risk subgroups of extensive-stage small cell lung cancer undergoing thoracic radiotherapy to improve overall survival in our next study.

The present work has overseen the development of the first prognostic model to predict the survival of patients with ES-SCLC and WHO PS 1-2 treated with TRT. Nevertheless, a number of limitations exist in this study. Firstly, the retrospective nature of the research may have introduced selection bias. Such a study design tends to lead to overestimation of the therapeutic effect, because there is a high likelihood that untreated patients are in a worse state than those given the option of treatment, despite this not always being recorded in the medical records. Furthermore, the heterogeneity of clinical parameters (e.g., a high percentage of young people) could have led to longer survival than that reported by randomized clinical trials. Our nomogram was built and subjected to internal validation; however, it should be externally validated in a large sample enrolled from multiple institutions. Besides, evaluation of 
the quality of life in these patients could potentially be a better reflection of the disease than the treatment received. Nonetheless, we carried out an in-depth real-world study which has preliminarily evidenced the potential value of serum inflammation markers to predict the prognosis of ESSCLC. Moreover, we put forward a hypothesis that requires validation by future prospective studies to determine the optimal cutoff for application in clinic.

In this research, we obtained data of 120 patients with symptomatic ES-SCLC, covering a period of 5 years. Concurrent TRT could prolong survival for symptomatic ES-SCLC patients. Integrating immunotherapy and TRT possibly augment the anti-tumor immune response and may potentiate a synergistic effect in locoregional control and enhance the immunotherapy effect on extrathoracic metastasis. Large-scale prospective cohort studies to put this treatment paradigm into practice for ES-SCLC are warranted. The results of our analysis indicate that NLR could potentially serve as a prognostic factor for mortality caused by ES-SCLC. Although dynamic monitoring of NLR over the course of treatment may have higher prognostic efficacy in ES-SCLC, we have demonstrated that NLR testing prior to TRT is also valuable for predicting the prognosis of frail ES-SCLC patients. The value of NLR as a predictive biomarker of immunotherapeutic efficacy warrants in-depth evaluative clinical research in the future. Additionally, we used peripheral blood markers to build a nomogram, which shows good discriminative power and calibration for the prediction of OS. This predictive model needs to be further validated in future prospective research.

\section{Acknowledgments}

The authors appreciate the academic support from AME Lung Cancer Collaborative Group, and thank professor Giorgio Vittorio Scagliotti for the critical comments and valuable advice on this study.

Funding: This work was supported by the National Natural Science Foundation of China (82072597 and 82003310).

\section{Footnote}

Reporting Checklist: The authors have completed the STROBE reporting checklist. Available at http://dx.doi. org/10.21037/tlcr-21-211

Data Sharing Statement: Available at http://dx.doi. org/10.21037/tlcr-21-211
Conflicts of Interest: All authors have completed the ICMJE uniform disclosure form (available at http://dx.doi. org/10.21037/tlcr-21-211). RB reports grants and other from AZ, BI, NOVARTIS, MSD, LILLY, ROCHE, Amgen, GSK, EISAI, outside the submitted work. The other authors have no conflicts of interest to declare.

Ethical Statement: The authors are accountable for all aspects of the work in ensuring that questions related to the accuracy or integrity of any part of the work are appropriately investigated and resolved. The study was conducted in accordance with the Declaration of Helsinki (as revised in 2013). The study was approved by the ethics board of Tongji Hospital of Tongji Medical College, Huazhong University of Science and Technology (TJIRB20190404). Individual consent for this retrospective analysis was waived.

Open Access Statement: This is an Open Access article distributed in accordance with the Creative Commons Attribution-NonCommercial-NoDerivs 4.0 International License (CC BY-NC-ND 4.0), which permits the noncommercial replication and distribution of the article with the strict proviso that no changes or edits are made and the original work is properly cited (including links to both the formal publication through the relevant DOI and the license). See: https://creativecommons.org/licenses/by-nc-nd/4.0/.

\section{References}

1. Gazdar AF, Bunn PA, Minna JD. Small-cell lung cancer: what we know, what we need to know and the path forward. Nat Rev Cancer 2017;17:765.

2. Rudin CM, Poirier JT. Small-cell lung cancer in 2016: Shining light on novel targets and therapies. Nat Rev Clin Oncol 2017;14:75-6.

3. Oronsky B, Reid TR, Oronsky A, Carter CA. What's New in SCLC? A Review. Neoplasia 2017;19:842-7.

4. Horn L, Mansfield AS, Szczęsna A, et al. First-Line Atezolizumab plus Chemotherapy in Extensive-Stage Small-Cell Lung Cancer. N Engl J Med 2018;379:2220-9.

5. Paz-Ares L, Dvorkin M, Chen Y, et al. Durvalumab plus platinum-etoposide versus platinum-etoposide in firstline treatment of extensive-stage small-cell lung cancer (CASPIAN): a randomised, controlled, open-label, phase 3 trial. Lancet 2019;394:1929-39.

6. Jeremic B, Shibamoto Y, Nikolic N, et al. Role of radiation therapy in the combined-modality treatment of patients 
with extensive disease small-cell lung cancer: A randomized study. J Clin Oncol 1999;17:2092-9.

7. Slotman BJ, van Tinteren H, Praag JO, et al. Use of thoracic radiotherapy for extensive stage small-cell lung cancer: a phase 3 randomised controlled trial. Lancet 2015;385:36-42.

8. Grivennikov SI, Greten FR, Karin M. Immunity, inflammation, and cancer. Cell 2010;140:883-99.

9. Wang D, Guo D, Shi F, et al. The predictive effect of the systemic immune-inflammation index for patients with small-cell lung cancer. Future Oncol 2019;15:3367-79.

10. Suzuki R, Lin SH, Wei X, et al. Prognostic significance of pretreatment total lymphocyte count and neutrophil-tolymphocyte ratio in extensive-stage small-cell lung cancer. Radiother Oncol 2018;126:499-505.

11. Berardi R, Rinaldi S, Santoni M, et al. Prognostic models to predict survival in patients with advanced non-small cell lung cancer treated with first-line chemo- or targeted therapy. Oncotarget 2016;7:26916-24.

12. Xie D, Marks R, Zhang M, et al. Nomograms Predict Overall Survival for Patients with Small-Cell Lung Cancer Incorporating Pretreatment Peripheral Blood Markers. J Thorac Oncol 2015;10:1213-20.

13. Li-Ming X, Zhao LJ, Simone CB 2nd, et al. Receipt of thoracic radiation therapy and radiotherapy dose are correlated with outcomes in a retrospective study of three hundred and six patients with extensive stage small-cell lung cancer. Radiother Oncol 2017;125:331-7.

14. Gao H, Dang Y, Qi T, et al. Mining prognostic factors of extensive-stage small-cell lung cancer patients using nomogram model. Medicine (Baltimore) 2020;99:e21798.

15. Bahij R, Jeppesen SS, Olsen KE, et al. Outcome of treatment in patients with small cell lung cancer in poor performance status. Acta Oncol 2019;58:1612-7.

16. Yee D, Butts C, Reiman A, et al. Clinical trial of postchemotherapy consolidation thoracic radiotherapy for extensive-stage small cell lung cancer. Radiother Oncol 2012;102:234-8.

17. Hasan S, Renz P, Turrisi A, et al. Dose escalation and associated predictors of survival with consolidative thoracic radiotherapy in extensive stage small cell lung cancer (SCLC): A National Cancer Database (NCDB) propensitymatched analysis. Lung Cancer 2018;124:283-90.

18. Han J, Fu C, Li B. Clinical outcomes of extensive-stage small cell lung cancer patients treated with thoracic radiotherapy at different times and fractionations. Radiat Oncol 2021;16:47.

19. Guo G, Wang Y, Zhou Y, et al. Immune cell concentrations among the primary tumor microenvironment in colorectal cancer patients predicted by clinicopathologic characteristics and blood indexes. J Immunother Cancer 2019;7:179

20. Ren X, Zhang Y, Lyu Y, et al. Lactate dehydrogenase and serum tumor markers for predicting metastatic status in geriatric patients with lung adenocarcinoma. Cancer Biomark 2019;26:139-50.

21. Wang Y, Qu X, Kam NW, et al. An inflammation-related nomogram for predicting the survival of patients with nonsmall cell lung cancer after pulmonary lobectomy. BMC Cancer 2018;18:692.

22. Amaral SR, Casal Moura M, Carvalho J, et al. 6PPrognostic significance of neutrophil-to-lymphocyte ratio (NLR) and platelet-to-lymphocyte ratio (PLR) in non-small cell lung cancer (NSCLC) treated with immune checkpoint inhibitors. Ann Oncol 2019. doi: 10.1093/ annonc/mdz027.004.

23. Diem S, Schmid S, Krapf M, et al. Neutrophil-toLymphocyte ratio (NLR) and Platelet-to-Lymphocyte ratio (PLR) as prognostic markers in patients with nonsmall cell lung cancer (NSCLC) treated with nivolumab. Lung Cancer 2017;111:176-81.

24. Passiglia F, Galvano A, Castiglia M, et al. Monitoring blood biomarkers to predict nivolumab effectiveness in NSCLC patients. Ther Adv Med Oncol 2019;11:1758835919839928.

25. Berardi R, Santoni M, Rinaldi S, et al. Pre-treatment systemic immune-inflammation represents a prognostic factor in patients with advanced non-small cell lung cancer. Ann Transl Med 2019;7:572.

Cite this article as: Yuan X, Zheng Z, Liu F, Gao Y, Zhang W, Berardi R, Mohindra P, Zhu Z, Lin J, Chu Q. A nomogram to predict the overall survival of patients with symptomatic extensive-stage small cell lung cancer treated with thoracic radiotherapy. Transl Lung Cancer Res 2021;10(5):2163-2171. doi: 10.21037/tlcr-21-211 


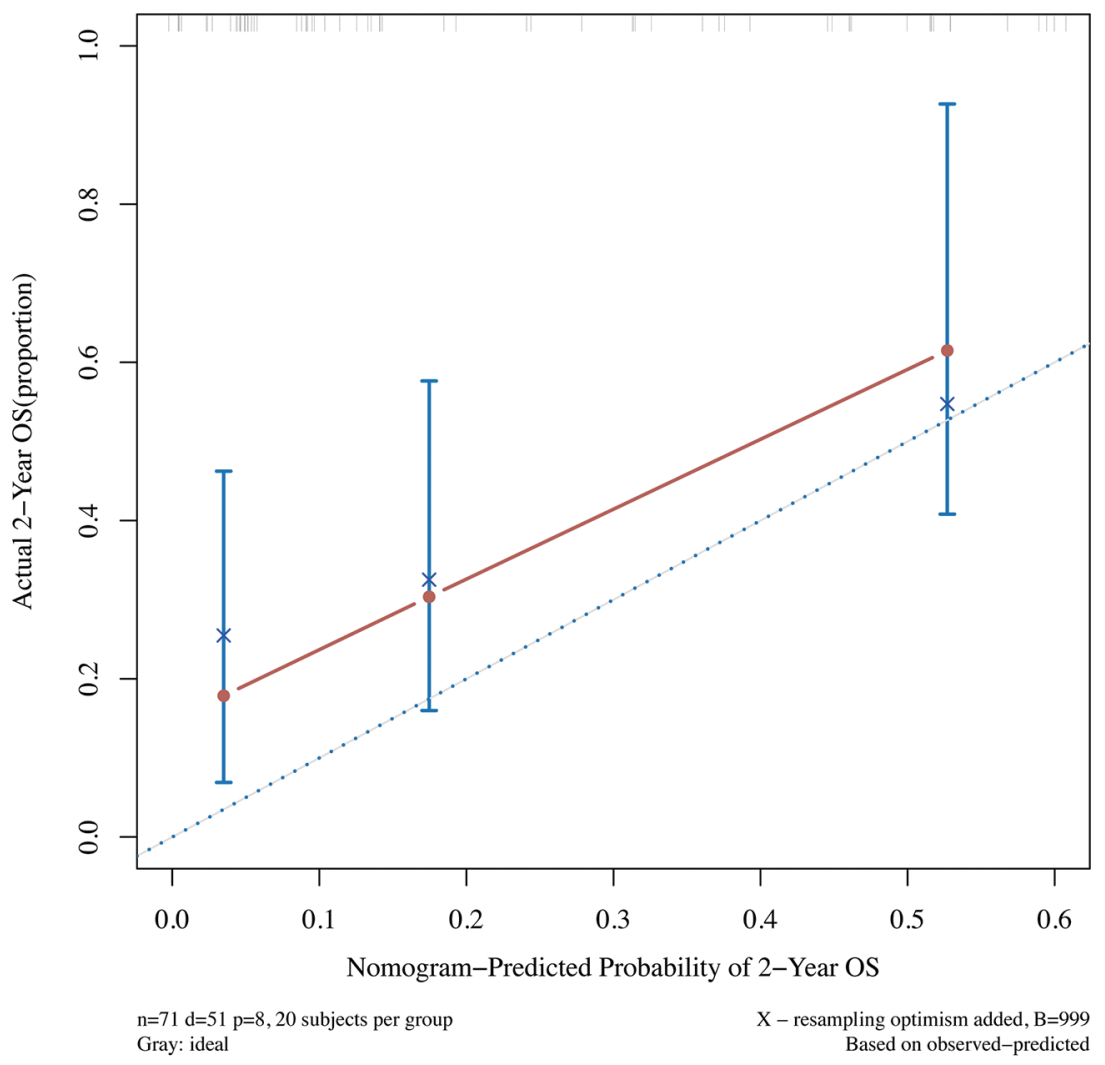

Figure S1 The nomogram's performance in patients with ES-SCLC. Calibration of 2-year OS predicted by the nomogram and that actually observed. On the calibration curve, the $\mathrm{x}$-axis is the probability of OS predicted using the nomogram, and the $\mathrm{y}$-axis is the observed OS. The reference line for an ideal nomogram is represented by the dotted line. The nomogram's performance is indicated by the solid line. The vertical lines represent $95 \%$ CIs. ES-SCLC, extensive-stage small cell lung cancer; OS, overall survival; CI, confidence interval. 